\title{
Wounded nucleon, quark, and quark-diquark emission functions versus experimental results from the BNL Relativistic Heavy Ion Collider at $\sqrt{s_{N N}}=200 \mathrm{GeV}$
}

\author{
Michał Barej, ${ }^{*}$ Adam Bzdak, ${ }^{\dagger}$ and Paweł Gutowski ${ }^{\ddagger}$ \\ AGH University of Science and Technology, Faculty of Physics and Applied Computer Science, 30-059 Kraków, Poland
}

(Received 11 April 2019; published 3 December 2019)

\begin{abstract}
Using the wounded nucleon, quark, and quark-diquark models, we extract the wounded source emission functions from the PHOBOS data for $d+\mathrm{Au}$ collisions at $\sqrt{s_{N N}}=200 \mathrm{GeV}$. We apply these functions to compute charged particle multiplicity distributions as functions of pseudorapidity for $p+p, p+\mathrm{Al}, p+\mathrm{Au}$, $d+\mathrm{Au},{ }^{3} \mathrm{He}+\mathrm{Au}, \mathrm{Cu}+\mathrm{Cu}, \mathrm{Cu}+\mathrm{Au}, \mathrm{Au}+\mathrm{Au}$, and $\mathrm{U}+\mathrm{U}$ collisions at the same energy and compare them with experimental data from the PHOBOS and PHENIX Collaborations. In symmetric collisions of heavy nuclei, the obtained distributions differ among the tested models. On the other hand, in asymmetric collisions, all three models give essentially the same distributions. The wounded quark-diquark and quark models are in reasonable agreement with data for all the investigated systems.
\end{abstract}

DOI: 10.1103/PhysRevC.100.064902

\section{INTRODUCTION}

In this paper, we examine various wounded source models commonly used to characterize the particle production in ultrarelativistic heavy-ion collisions. In the wounded nucleon model (WNM) [1], a heavy ion collision is described as a superposition of multiple independent nucleon-nucleon interactions. The wounded quark model (WQM) [2] is similar but constituent quark-quark collisions are considered. This model was studied recently in various contexts with rather interesting results [3-13]. In the wounded quark-diquark model (WQDM) [14], a nucleon is assumed to consist of a quark and a diquark. This model not only reproduces charge particle multiplicities but also naturally explains the differential elastic cross section in proton-proton collisions in a broad range of energies [14-19]. In all three wounded source models, it is assumed that every constituent (nucleon, quark, or diquark, depending on the model) which underwent at least one inelastic collision produces particles independently of the number of collisions. Such sources we call wounded.

As explained in detail in Ref. [14], the wounded constituent model may be applicable to particles with not too large transverse masses. In this case, the particle formation time is large enough and the individual collisions cannot be resolved, leading to the wounded constituent scaling (in contrast to the number of collisions scaling).

\footnotetext{
*michal.barej@fis.agh.edu.pl

†adam.bzdak@fis.agh.edu.pl

†pawel.gutowski@fis.agh.edu.pl
}

Published by the American Physical Society under the terms of the Creative Commons Attribution 4.0 International license. Further distribution of this work must maintain attribution to the author(s) and the published article's title, journal citation, and DOI. Funded by SCOAP .
In the wounded constituent models, it is usually assumed that the number of final particles is solely driven by the initial stage entropy decomposition. This assumption might sound a bit extreme; however, various results (including the extensive analysis of particle multiplicities in the wounded quark model by the PHENIX Collaboration for a broad range of energies $[3,4]$ ) suggest that possibly it is not that far from reality.

In our previous work [12], we investigated the wounded quark emission function $F(\eta)$ extracted from $d+{ }^{197} \mathrm{Au}$ collisions at $\sqrt{s_{N N}}=200 \mathrm{GeV}$ measured by the PHOBOS Collaboration at the Relativistic Heavy Ion Collider (RHIC) [20]. This function is defined as the pseudorapidity particle multiplicity distribution from one wounded source. We observed that the wounded-quark emission function, $F(\eta)$, is practically universal in various centrality classes; see also Ref. [13]. Using minimum bias (min-bias) $F(\eta)$, we predicted the pseudorapidity charged particle multiplicity distributions, $d N_{\mathrm{ch}} / d \eta$, for $p+{ }^{27} \mathrm{Al}, p+{ }^{197} \mathrm{Au}$, and ${ }^{3} \mathrm{He}+$ ${ }^{197} \mathrm{Au}$. Our predictions turned out to be in good agreement with recent experimental results from the PHENIX Collaboration [21].

In this paper, we study three different emission functions extracted from the WNM, WQM, and WQDM. Using these functions we compute $d N_{\mathrm{ch}} / d \eta$ distributions in various centrality classes at $\sqrt{s_{N N}}=200 \mathrm{GeV}$ for several colliding systems measured by PHOBOS and PHENIX. Our goal is to determine which model is best able to reproduce the experimental results.

As expected, for symmetric collisions such as ${ }^{63} \mathrm{Cu}+{ }^{63} \mathrm{Cu}$ or ${ }^{197} \mathrm{Au}+{ }^{197} \mathrm{Au}$ there are significant differences among the studied models. Namely, the wounded nucleon model is unsuitable for these collisions (except very peripheral ones), whereas both the wounded quark and quark-diquark models are in good agreement with the RHIC results on $d N_{\mathrm{ch}} / d \eta$ $[22,23]$. On the other hand, in asymmetric collisions (such as the ones studied in, e.g., Ref. [21]) with one light nucleus ( $p$, $d$, and ${ }^{3} \mathrm{He}$ ) all three models give practically identical results. 
We also made simulations for ${ }^{63} \mathrm{Cu}+{ }^{197} \mathrm{Au}$ and ${ }^{238} \mathrm{U}+{ }^{238} \mathrm{U}$ and compared them with available data from PHENIX [4].

This paper is organized as follows. In the next section, there is a brief description of the wounded source models. Next, we show the minimum-bias emission functions extracted from the PHOBOS data for $d+{ }^{197}$ Au collisions. Finally, we compare our simulations with the RHIC results for nucleusnucleus collisions at $\sqrt{s_{N N}}=200 \mathrm{GeV}$. In the last section, our conclusions are presented.

\section{WOUNDED SOURCE MODELS}

We consider three models which differ by the composition of a nucleus; i.e., a nucleus consists of nucleons in the WNM (no internal structure), constituent quarks and diquarks in the WQDM, and constituent quarks in the WQM. In all three models we assume that every wounded constituent populates charged particles regardless of the number of collisions [1]. Therefore, we can treat a collision of two nuclei as a superposition of independent nucleon-nucleon, quark-quark, or quark(diquark)-quark(diquark) interactions. In general, the pseudorapidity multiplicity distribution of charged particles is given by [24]

$$
\frac{d N_{\mathrm{ch}}}{d \eta}=w_{L} F(\eta)+w_{R} F(-\eta),
$$

where $F( \pm \eta)$ is the emission function of one constituent (nucleon, quark, or diquark) and the coefficients $w_{L}, w_{R}$ are the mean numbers of wounded constituents in the left-going and the right-going nuclei, respectively. $w_{L}$ and $w_{R}$ are calculated using the Glauber Monte Carlo simulations with parameters listed in Ref. [25]. The detailed explanation of our simulation is presented below. If $w_{L} \neq w_{R}$ we can extract $F(\eta)$ :

$$
F(\eta)=\frac{1}{2}\left[\frac{N(\eta)+N(-\eta)}{w_{L}+w_{R}}+\frac{N(\eta)-N(-\eta)}{w_{L}-w_{R}}\right],
$$

where $N( \pm \eta)=d N_{\mathrm{ch}}( \pm \eta) / d \eta$. We emphasize here that using symmetric collisions, such as proton-proton, for which $w_{L}=$ $w_{R}$, one can extract $F(\eta)+F(-\eta)$ only; see Eq. (1). In order to extract $F(\eta)$ one needs asymmetric collisions characterized by $w_{L} \neq w_{R}$; see the second term of Eq. (2). ${ }^{1}$

The positions of nucleons in nuclei are randomly drawn from the appropriate distributions. For the deuteron, the Hulthen formula determines the proton's position:

$$
\rho(\vec{r})=\rho_{0}\left(\frac{e^{-A r}+e^{-B r}}{r}\right)^{2},
$$

where $r$ is a distance from the center of a nucleus with parameters $A=0.457 \mathrm{fm}^{-1}, B=2.35 \mathrm{fm}^{-1}$ and the neutron is placed opposite to the proton $[25,26]$. For helium-3 we used [27] to determine the nucleons' coordinates $\vec{r}$. For gold,

\footnotetext{
${ }^{1} \mathrm{~A}$ possible interpretation of the two terms present in Eq. (2), identifying one component with the gluon cloud and another one with the valence part, is given in Ref. [24].
}

TABLE I. Parameters used in our calculations for the WoodsSaxon distribution; see Eq. (4).

\begin{tabular}{lcccc}
\hline \hline & $a(\mathrm{fm})$ & $R(\mathrm{fm})$ & $\beta_{2}$ & $\beta_{4}$ \\
\hline${ }^{27} \mathrm{Al}$ & 0.580 & 3.34 & -0.448 & 0.239 \\
${ }^{63} \mathrm{Cu}$ & 0.596 & 4.20 & 0 & 0 \\
${ }^{197} \mathrm{Au}$ & 0.535 & 6.38 & 0 & 0 \\
${ }^{238} \mathrm{U}$ & 0.440 & 6.67 & 0.280 & 0.093 \\
\hline \hline
\end{tabular}

copper, aluminium, and uranium, the positions of nucleons are given by the Woods-Saxon distribution

$$
\rho(\vec{r})=\rho_{0}\left[1+\exp \left(\frac{r-R\left(1+\beta_{2} Y_{20}+\beta_{4} Y_{40}\right)}{a}\right)\right]^{-1},
$$

where $\quad Y_{20}=\sqrt{\frac{5}{16 \pi}}\left(3 \cos ^{2} \theta-1\right), \quad Y_{40}=\frac{3}{16 \sqrt{\pi}}\left(35 \cos ^{4} \theta-\right.$ $\left.30 \cos ^{2} \theta+3\right)$, and all the parameters are listed in Table I [25,28]. For the WQM we generate three quarks independently according to [29]

$$
\rho(\vec{r})=\rho_{0} \exp \left(-\sqrt{12} C r / r_{p}\right),
$$

with $r_{p}=0.81 \mathrm{fm}$ being the proton's radius and the coefficient $C=0.82$ results from shifting the quarks to the center of mass of a nucleon. ${ }^{2}$ In the WQDM, we generate a quark at a distance $r$ from the center of mass according to Eq. (5) with $C=0.79$ and then place a diquark in the opposite direction at a distance of $r / 2$. This is, of course, equivalent to the assumption that a diquark is two times heavier than a quark. We verified that this assumption is not crucial in our calculations. Assuming for example that both masses are equal, we obtained almost identical results.

In the next step, we draw the squared impact parameter $b^{2}$ from a uniform distribution in an interval of $\left[0, b_{\max }^{2}\right]$. We took $b_{\max }$ to be $5 \mathrm{fm}$ for $p+p ; 9 \mathrm{fm}$ for $p+\mathrm{Al}$; $15 \mathrm{fm}$ for $p+\mathrm{Au}, d+\mathrm{Au},{ }^{3} \mathrm{He}+\mathrm{Au}, \mathrm{Cu}+\mathrm{Cu} ; 18 \mathrm{fm}$ for $\mathrm{Cu}+\mathrm{Au}, \mathrm{Au}+\mathrm{Au}$; and $20 \mathrm{fm}$ for $\mathrm{U}+\mathrm{U}$ collisions. Then we count wounded sources by checking whether each source from one nucleus collided with at least one source from another one. To determine if two constituents interact with each other we used a normal distribution and checked whether the transverse distance $s$ between colliding sources and the random variable $u$ (from a uniform distribution in $[0,1])$ satisfy $u<\exp \left(-s^{2} / 2 \gamma^{2}\right)$, where $\gamma^{2}=\sigma_{\text {ii }} / 2 \pi$ and $\sigma_{\text {ii }}$ is an inelastic constituent-constituent cross section. For collisions at $\sqrt{s_{N N}}=200 \mathrm{GeV}$ we took the corresponding nucleon-nucleon inelastic cross section of $\sigma_{\mathrm{nn}}=41 \mathrm{mb}$ [25]. For the WQM we determined $\sigma_{\mathrm{qq}}$ using the trial-and-error method [12]. We found the value of $\sigma_{\mathrm{qq}}=6.65 \mathrm{mb}$, which satisfies $\sigma_{\mathrm{nn}}=\int_{0}^{2 \pi} d \varphi \int_{0}^{+\infty} d s s P(s)$, where $P(s)$ is the probability of the inelastic collision of two nucleons with the transverse distance $s$. In the WQDM there are three possible types of collisions of constituents: quark-quark, quark-diquark, and diquark-diquark. With this in mind,

\footnotetext{
${ }^{2}$ We choose the parameter $C$ in Eq. (5) so that $\left\langle r^{2}\right\rangle=r_{p}^{2}$ for generated quarks or quarks and diquarks.
} 

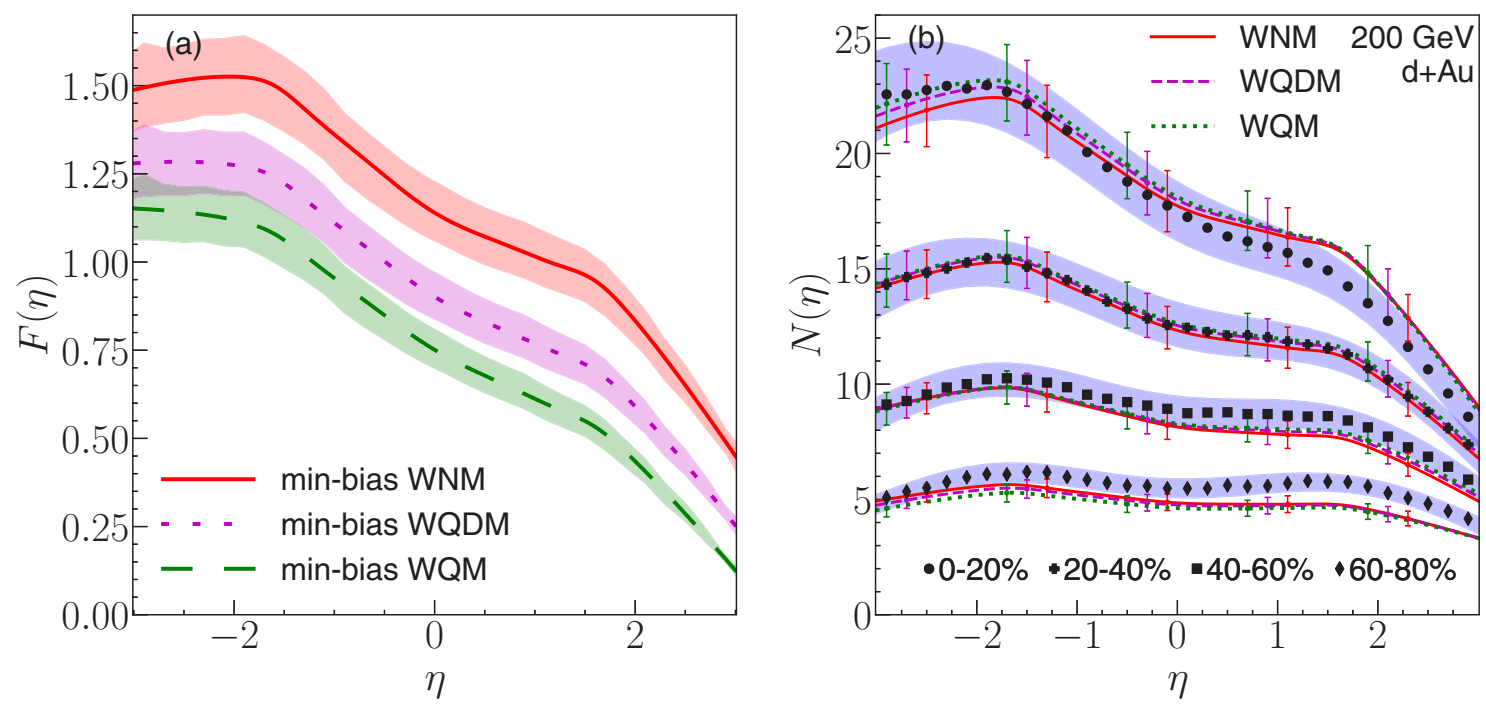

FIG. 1. (a) The min-bias wounded nucleon, quark-diquark, and quark emission functions at $\sqrt{s_{N N}}=200 \mathrm{GeV}$. (b) Reconstruction of charged particle multiplicity distributions $N(\eta) \equiv d N / d \eta$ as functions of pseudorapidity for $d+{ }^{197}$ Au using the calculated min-bias emission functions. Points represent the PHOBOS data and lines represent our simulation results. Shaded areas and bars represent corresponding uncertainties for the PHOBOS data and our calculations, respectively.

we modified our trial-and-error procedure for the WQDM assuming that the corresponding cross sections satisfy the proportion $\sigma_{\mathrm{qq}}^{\prime}: \sigma_{\mathrm{qd}}: \sigma_{\mathrm{dd}}=1: 2: 4$ [14]. Using the trial-and-error method we found that $\sigma_{\mathrm{qq}}^{\prime}=5.75 \mathrm{mb}$.

To calculate the number of emitted charged particles (used, e.g., to determine the centrality class) we assumed that each wounded source populates particles according to a negative binomial distribution, described by two parameters $\langle n\rangle$ and $k$, the latter characterizing the deviation from a Poisson distribution. In the WNM we took $\langle n\rangle=5$ and $k=1$ [30]. For the WQM and the WQDM these numbers should be divided by the mean numbers of wounded constituents in a nucleonnucleon collision, which are equal to 1.27 (WQM) and 1.14 (WQDM) per one nucleon.

\section{EMISSION FUNCTIONS}

Our goal is to extract the wounded source emission functions from the minimum-bias $d+$ Au collisions measured by PHOBOS. Next, using Eq. (1) we can calculate $d N_{\mathrm{ch}} / d \eta$ for all colliding systems and all centralities of interest. We note that this procedure does not introduce any free parameters (the only parameters we use are the ones from the Woods-Saxon distribution, etc.)

Performing the Glauber Monte Carlo calculations, described in the previous section, we determined the mean numbers of wounded constituents, $w_{L}$ and $w_{R}$ [see Eq. (1)], in min-bias $d+{ }^{197} \mathrm{Au}$ collisions; see Table II (where we also show the results for other measured centralities). With these values and the PHOBOS data, the min-bias emission functions for wounded nucleons, quarks, and diquarks are calculated according to Eq. (2) and presented in Fig. 1(a). We note that in this calculation we fitted the PHOBOS data with an analytical function, as described in the Appendix. In Fig. 1(b) we show how our models reproduce the data for $d+{ }^{197} \mathrm{Au}$ at $\sqrt{s_{N N}}=200 \mathrm{GeV}$, based on the min-bias emission functions. ${ }^{3}$ One can see that all models describe the data quite well, although certain discrepancies can be observed. We also note that all models give practically identical results.

\section{RESULTS}

\section{$\mathrm{A} . p+\mathrm{Al}, p+\mathrm{Au}, d+\mathrm{Au}$, and $\mathrm{He}+\mathrm{Au}$}

First, we compare our simulations in all three models with the recent PHENIX results for asymmetric collisions at $\sqrt{s_{N N}}=200 \mathrm{GeV}$ [21]. In Tables III-VI we show the mean numbers of wounded sources, $w_{L}$ and $w_{R}$ [see Eq. (1)], in $p+{ }^{27} \mathrm{Al}, p+{ }^{197} \mathrm{Au}, d+{ }^{197} \mathrm{Au}$, and ${ }^{3} \mathrm{He}+{ }^{197} \mathrm{Au}$ collisions for different centrality classes. In Figures $2-5$ we

${ }^{3}$ In Fig. 1(b) we do not show the min-bias points since they are described in our models by construction.

TABLE II. The mean numbers of wounded nucleons, quark-diquarks, and quarks, $w_{L}$ and $w_{R}$ as seen in Eq. (1), for various centrality classes in $d+{ }^{197} \mathrm{Au}$ collisions at $\sqrt{s_{N N}}=200 \mathrm{GeV}$. The first and second numbers in each cell concern $d$ and Au, respectively.

\begin{tabular}{|c|c|c|c|c|c|}
\hline & min-bias & 0-20\% & $20-40 \%$ & $40-60 \%$ & $60-80 \%$ \\
\hline WNM & $1.60,6.56$ & $1.97,13.59$ & $1.86,8.96$ & $1.65,5.49$ & $1.36,2.90$ \\
\hline WQDM & $2.65,7.67$ & $3.80,16.15$ & $3.39,10.52$ & $2.74,6.40$ & $1.95,3.33$ \\
\hline
\end{tabular}


TABLE III. The mean numbers of wounded nucleons, quark-diquarks, and quarks, $w_{L}$ and $w_{R}$ as seen in Eq. (1), for different centrality classes in $p+{ }^{27} \mathrm{Al}$ collisions at $\sqrt{s_{N N}}=200 \mathrm{GeV}$. The first and second numbers in each cell concern $p$ and Al, respectively.

\begin{tabular}{lccccc}
\hline \hline & min-bias & $0-5 \%$ & $5-10 \%$ & $10-20 \%$ & $20-40 \%$ \\
\hline WNM & $1.00,1.96$ & $1.00,3.85$ & $1.00,3.10$ & $1.00,2.66$ & $1.00,2.17$ \\
WQDM & $1.40,2.25$ & $1.85,4.77$ & $1.76,3.81$ & $1.67,3.23$ & $1.53,2.55$ \\
WQM & $1.76,2.51$ & $2.68,5.61$ & $2.51,4.49$ & $2.33,3.78$ & $2.03,2.92$ \\
\hline \hline
\end{tabular}

TABLE IV. Same as Table III but for $p+{ }^{197} \mathrm{Au}$ collisions.

\begin{tabular}{|c|c|c|c|c|c|c|c|}
\hline WNM & $1.00,4.47$ & $1.00,10.07$ & $1.00,8.52$ & $1.00,7.35$ & $1.00,5.68$ & $1.00,3.93$ & $1.00,2.44$ \\
\hline WQDM & $1.66,5.11$ & $1.99,11.84$ & $1.98,9.93$ & $1.95,8.51$ & $1.89,6.59$ & $1.75,4.57$ & $1.46,2.69$ \\
\hline
\end{tabular}

TABLE V. Same as Table III but for $d+{ }^{197}$ Au collisions.

\begin{tabular}{|c|c|c|c|c|c|c|c|}
\hline WNM & $1.59,6.56$ & $1.99,16.48$ & $1.98,13.96$ & $1.95,11.97$ & $1.86,8.96$ & $1.65,5.49$ & $1.31,2.62$ \\
\hline WQDM & $2.65,7.67$ & $3.92,19.75$ & $3.84,16.55$ & $3.72,14.13$ & $3.39,10.52$ & $2.74,6.40$ & $1.81,2.93$ \\
\hline
\end{tabular}

TABLE VI. Same as Table III but for ${ }^{3} \mathrm{He}+{ }^{197} \mathrm{Au}$ collisions.

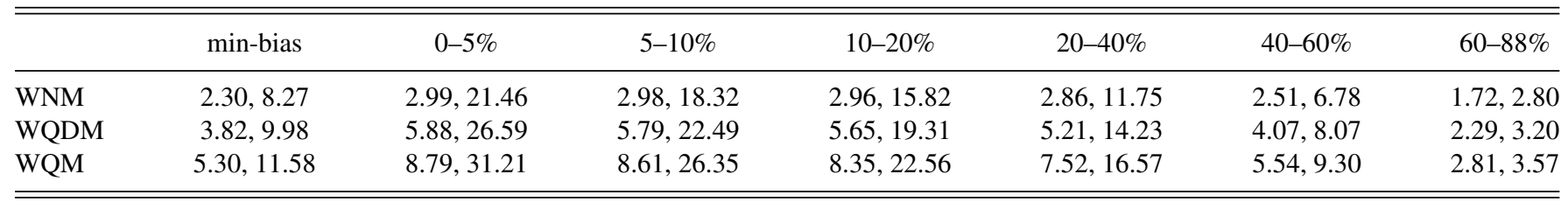

TABLE VII. The mean numbers of wounded nucleons, quark-diquarks, and quarks per one nucleus [in symmetric collisions $w_{L}=w_{R}$ in Eq. (1)] for different centrality classes in ${ }^{63} \mathrm{Cu}+{ }^{63} \mathrm{Cu}$ collisions at $\sqrt{s_{N N}}=200 \mathrm{GeV}$.

\begin{tabular}{lcccccrr}
\hline \hline & min-bias & $0-6 \%$ & $6-15 \%$ & $15-25 \%$ & $25-35 \%$ & $35-45 \%$ & $45-55 \%$ \\
\hline WNM & 16.2 & 51.6 & 42.2 & 31.2 & 22.0 & 15.0 & 9.9 \\
WQDM & 24.7 & 87.1 & 67.7 & 48.1 & 32.5 & 21.4 & 13.5 \\
WQM & 32.5 & 121.0 & 91.6 & 63.2 & 41.8 & 26.7 & 16.5 \\
\hline \hline
\end{tabular}

TABLE VIII. Same as Table VII but for ${ }^{197} \mathrm{Au}+{ }^{197} \mathrm{Au}$ collisions.

\begin{tabular}{|c|c|c|c|c|c|c|c|}
\hline & min-bias & $0-6 \%$ & $6-15 \%$ & $15-25 \%$ & $25-35 \%$ & $35-45 \%$ & $45-55 \%$ \\
\hline WNM & 50.2 & 172.7 & 136.3 & 98.6 & 68.9 & 46.1 & 29.1 \\
\hline WQDM & 83.6 & 313.1 & 237.5 & 165.4 & 110.4 & 71.1 & 42.9 \\
\hline
\end{tabular}




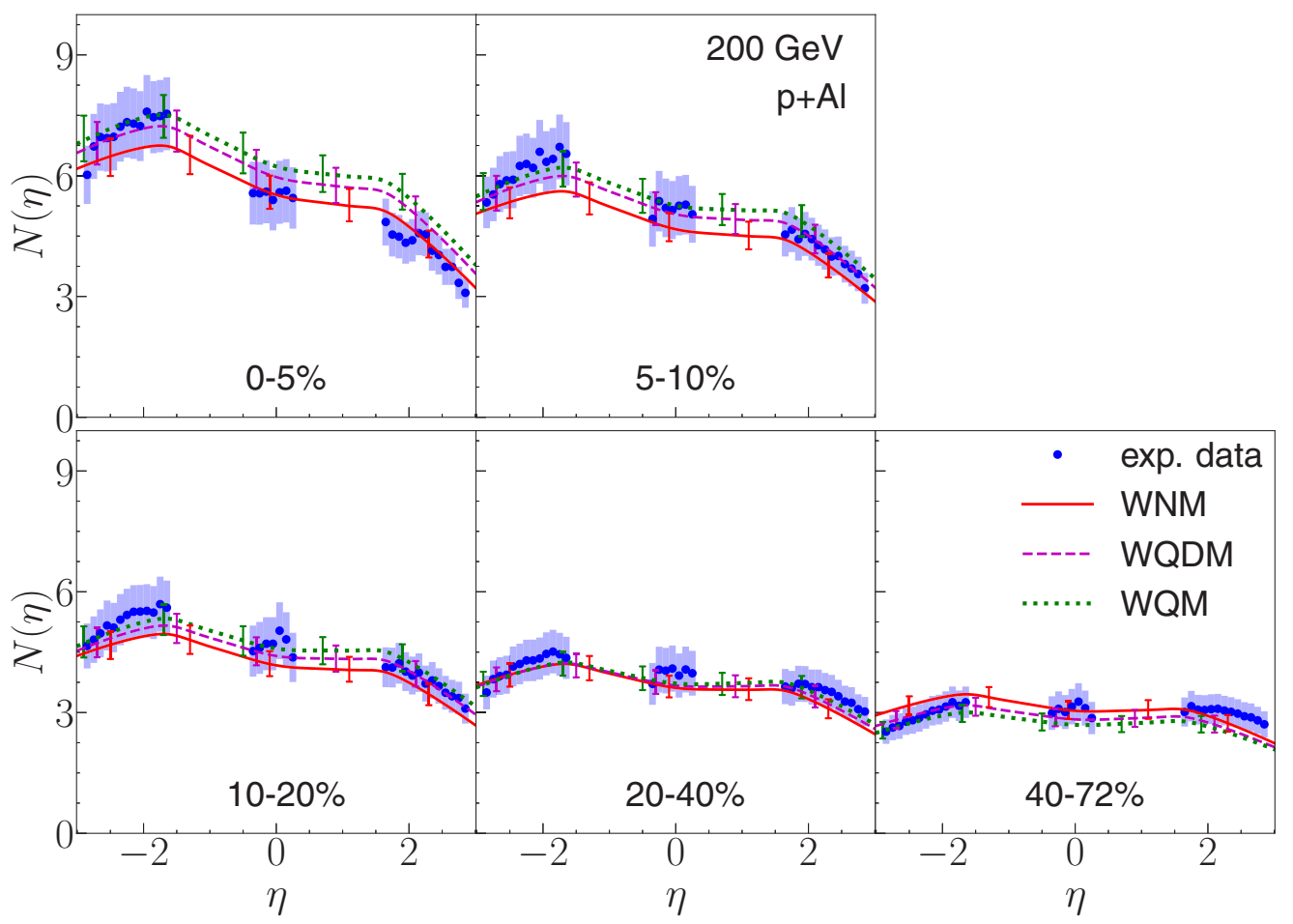

FIG. 2. Charged particle multiplicity distribution $N(\eta)$ as a function of pseudorapidity in the WNM (wounded nucleon model), the WQDM (wounded quark-diquark model), and the WQM (wounded quark model) in various centrality classes for $p+{ }^{27} \mathrm{Al}$ collision at $\sqrt{s_{N N}}=200 \mathrm{GeV}$. Dots represent the PHENIX data [21]. Uncertainties are marked as bars for our simulation and as shaded areas for the experiment. Note that the measurement was carried out in the limited ranges of $\eta$.

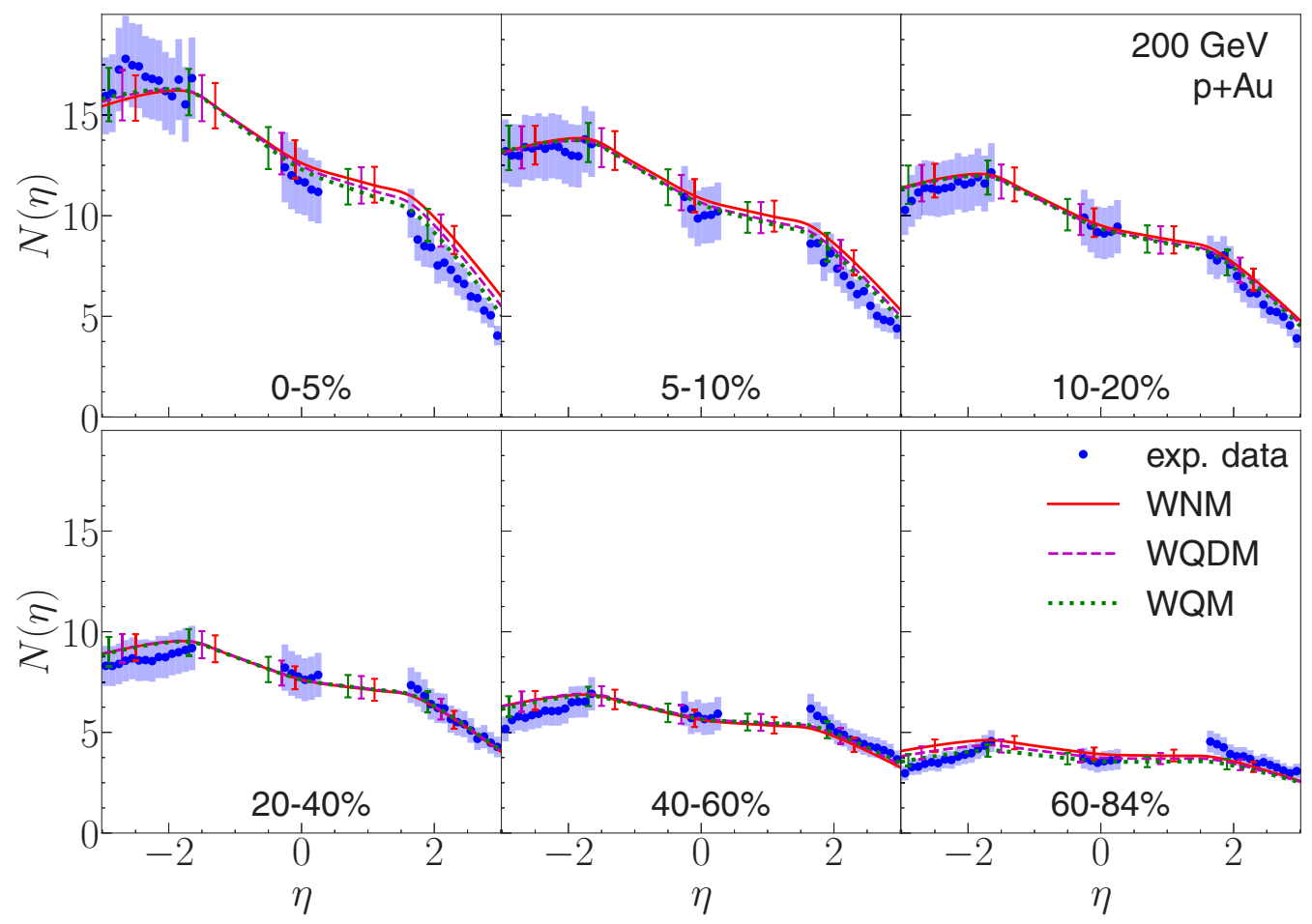

FIG. 3. Same as Figure 2 but for $p+{ }^{197}$ Au collision. 


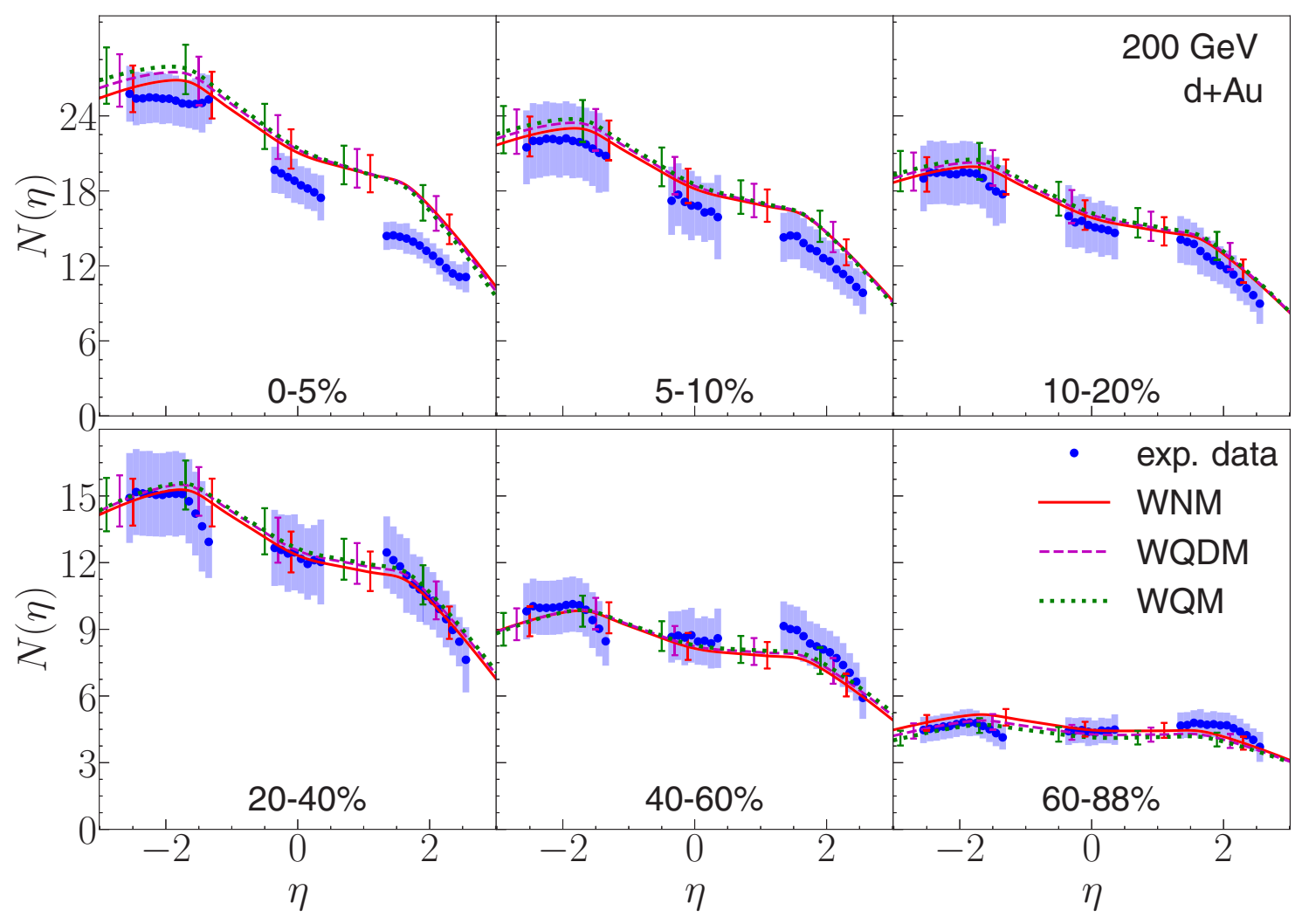

FIG. 4. Same as Figure 2 but for $d+{ }^{197}$ Au collision.

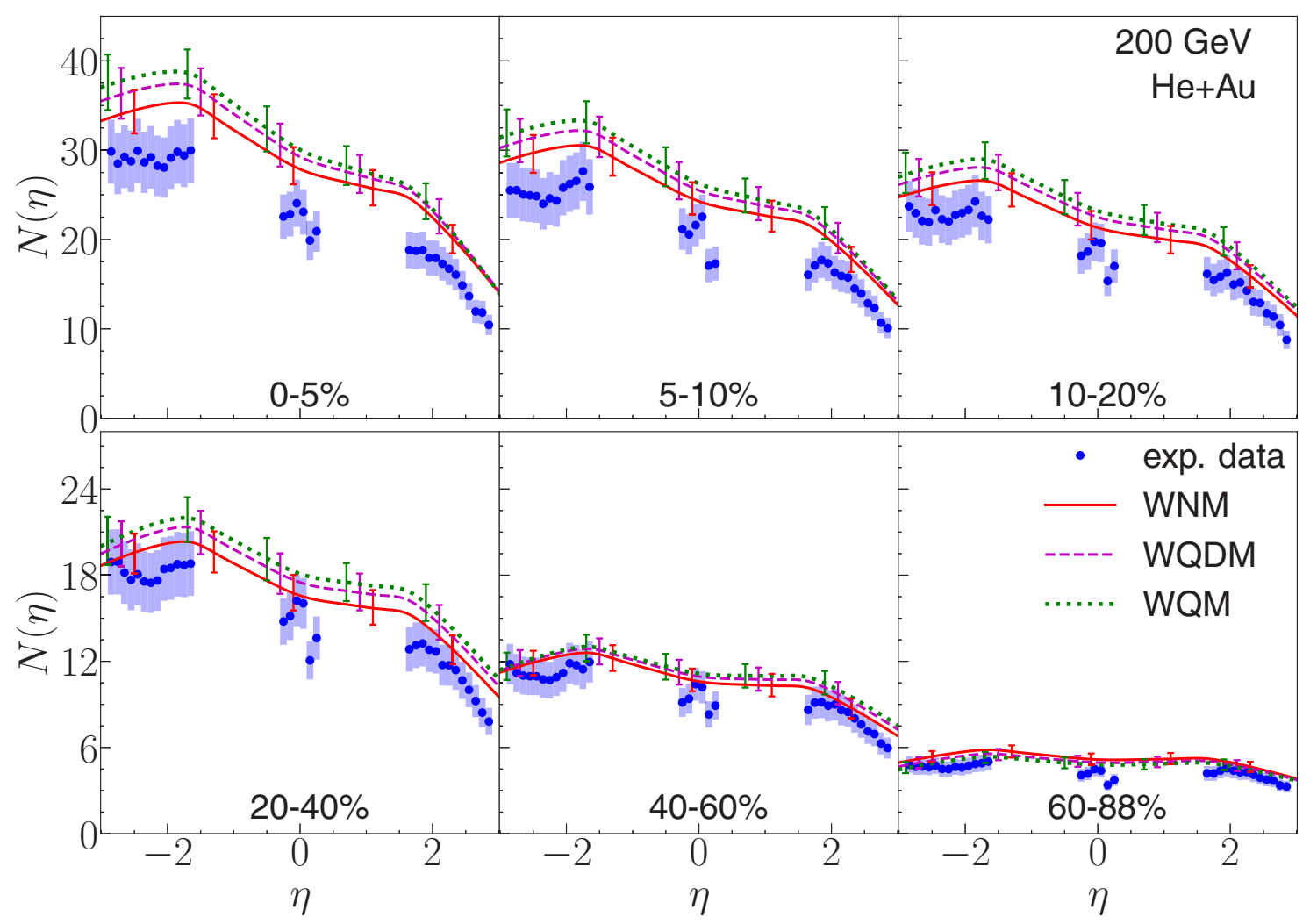

FIG. 5. Same as Fig. 2 but for ${ }^{3} \mathrm{He}+{ }^{197} \mathrm{Au}$ collision. 


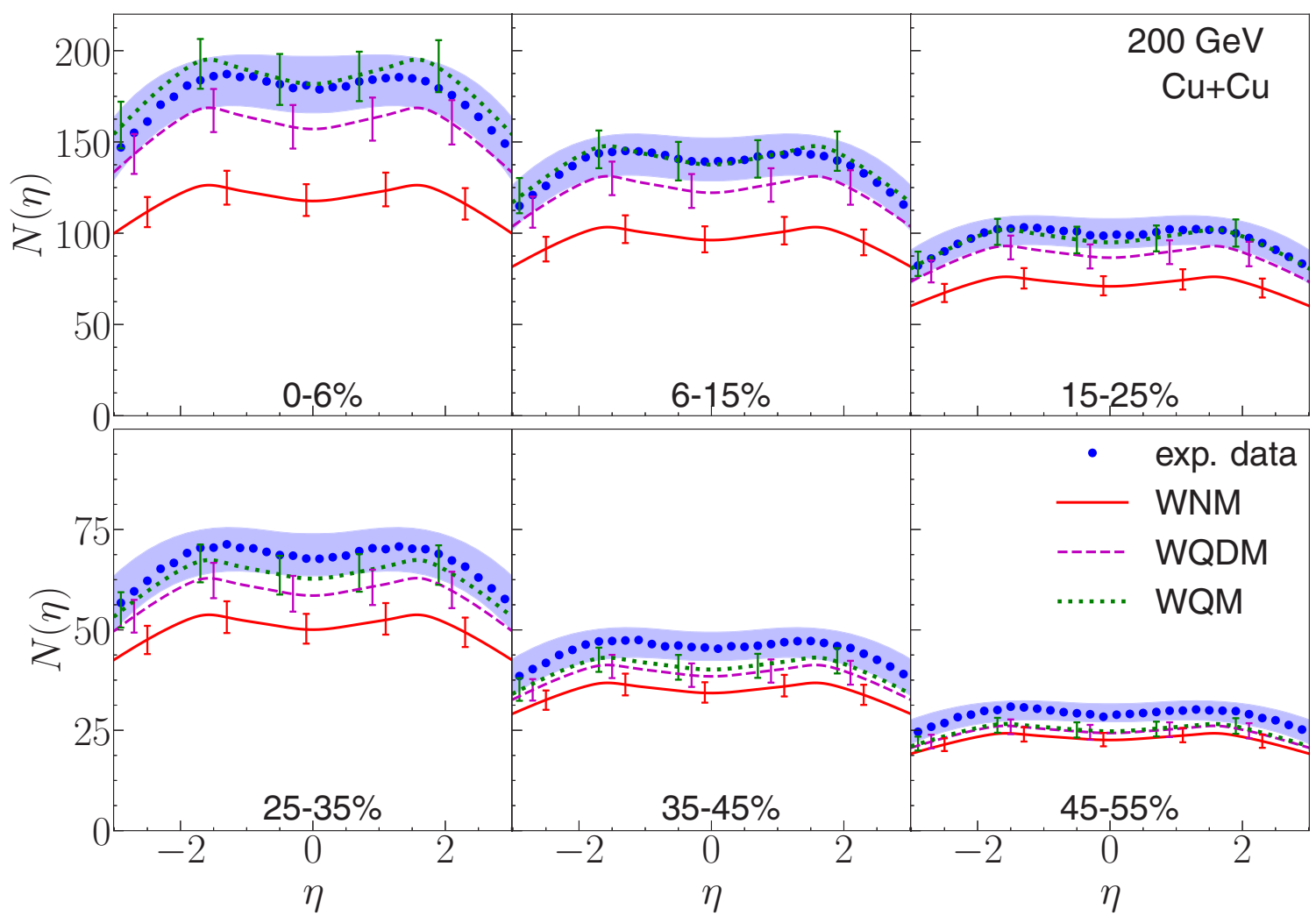

FIG. 6. Same as Figure 2 but for ${ }^{63} \mathrm{Cu}+{ }^{63} \mathrm{Cu}$ collisions. Dots represent the PHOBOS data [22].

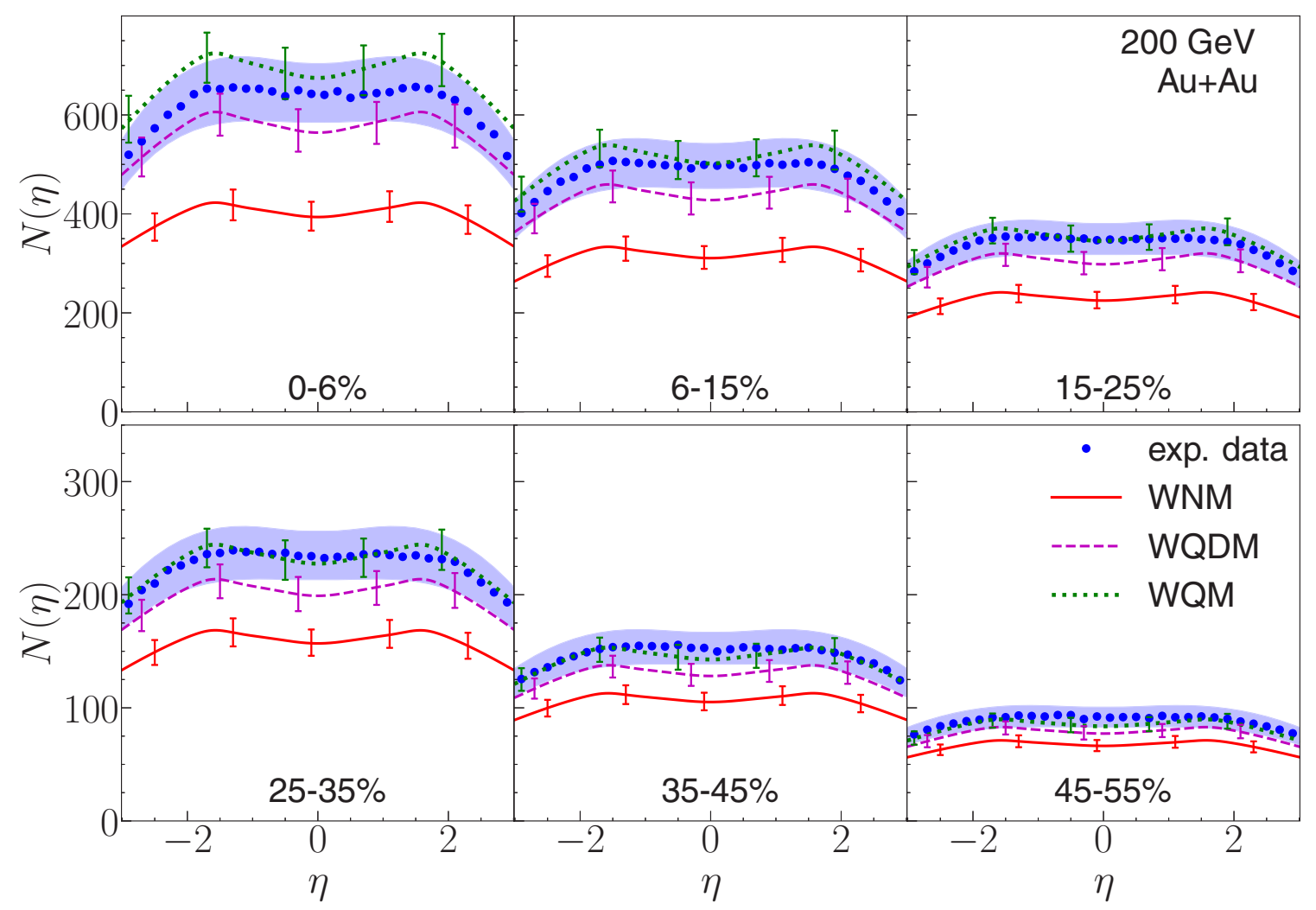

FIG. 7. Same as Figure 2 but for ${ }^{197} \mathrm{Au}+{ }^{197} \mathrm{Au}$ collisions. Dots represent the PHOBOS data [23]. 
TABLE IX. Same as Table III but for ${ }^{63} \mathrm{Cu}+{ }^{197} \mathrm{Au}$ collisions.

\begin{tabular}{|c|c|c|c|c|c|c|c|}
\hline & min-bias & $0-5 \%$ & $5-10 \%$ & $15-20 \%$ & $25-30 \%$ & $35-40 \%$ & $45-50 \%$ \\
\hline WQDM & $36.4,52.6$ & $115.6,205.9$ & $105.5,168.9$ & $80.5,114.2$ & $56.9,75.1$ & $38.0,47.9$ & $24.1,29.4$ \\
\hline
\end{tabular}

present the calculated pseudorapidity charged particle multiplicity distributions. We note that our predictions based on the WQM, published in Ref. [12], have already been successfully verified by PHENIX; see Ref. [21].

In the above figures one can observe that the differences between the models are negligible for the studied asymmetric collisions. All simulations are in quite good agreement with the results from the PHENIX Collaboration [21]. The largest disagreement (of the order of $20 \%$ ) is for the most central ${ }^{3} \mathrm{He}+\mathrm{Au}$ collisions.

We note that $F(\eta)$ is extracted from the min-bias PHOBOS data on $d+{ }^{197} \mathrm{Au}$ collisions and all our calculations are basically parameter free. Consequently, one should not expect to obtain a better agreement than a few tens of percent.

\section{B. $\mathrm{Cu}-\mathrm{Cu}$ and $\mathrm{Au}-\mathrm{Au}$}

Here we test all three models with the PHOBOS results on $d N_{\mathrm{ch}} / d \eta$ for ${ }^{63} \mathrm{Cu}+{ }^{63} \mathrm{Cu}$ and ${ }^{197} \mathrm{Au}+{ }^{197} \mathrm{Au}$ collisions at $\sqrt{s_{N N}}=200 \mathrm{GeV}$. Tables VII and VIII contain the mean numbers of wounded sources (per one nucleus since $w_{L}=w_{R}$ for $\mathrm{Cu}+\mathrm{Cu}$ and $\mathrm{Au}+\mathrm{Au}$ ) for these collisions and Figs. 6 and 7 demonstrate the calculated and measured pseudorapidity charged particle multiplicity distributions.

In the case of symmetric collisions, we get distributions which differ significantly among the models. As expected, the wounded nucleon model is not valid for central collisions of heavy nuclei. On the other hand, our results from the wounded quark and quark-diquark models are in quite good agreement with the data.

\section{C. $\mathrm{Cu}-\mathrm{Au}$ and $\mathrm{U}-\mathrm{U}$}

Next, we discuss ${ }^{63} \mathrm{Cu}+{ }^{197} \mathrm{Au}$ and ${ }^{238} \mathrm{U}+{ }^{238} \mathrm{U}$ collisions where we have very limited experimental data to compare with [4]. The mean numbers of wounded sources, $w_{L}$ and $w_{R}$, are presented in Tables IX and $\mathrm{X}$ and the obtained distributions $d N_{\mathrm{ch}} / d \eta$ in Figs. 8 and 9. For these collisions, we show only a few selected centrality bins.

Results for ${ }^{63} \mathrm{Cu}+{ }^{197} \mathrm{Au}$ and ${ }^{238} \mathrm{U}+{ }^{238} \mathrm{U}$ again indicate that the WQDM and the WQM are in acceptable agreement with the experimental data. In central ${ }^{63} \mathrm{Cu}+$ ${ }^{197} \mathrm{Au}$ collisions WQDM seems to be closer to the data; however, it is rather difficult to draw any definite conclusions with the admittedly large error bars and a single data point per centrality; see Fig. 8. We note that in the case of ${ }^{238} \mathrm{U}+{ }^{238} \mathrm{U}$ collisions, the collision energy is $\sqrt{s_{N N}}=193 \mathrm{GeV}$ and in our simulations we used the wounded constituent emission functions extracted at $\sqrt{s_{N N}}=200 \mathrm{GeV}$. It is rather obvious that this small difference in energy is negligible at our accuracy level.

\section{D. $p+p$}

Lastly, we present our calculations for proton-proton collisions. The obtained mean numbers of wounded sources, per one wounded proton, are 1.00,1.14, and 1.27 for the wounded nucleon, quark-diquark, and quark models, respectively. Figure 10 shows the calculated and measured by PHOBOS pseudorapidity charged particle multiplicity distributions.

As seen in Fig. 10, in $p+p$ collisions all models agree (within uncertainties) with the data.

\section{DISCUSSION AND CONCLUSIONS}

The goal of our paper was to study all colliding systems measured at RHIC at $\sqrt{s_{N N}}=200 \mathrm{GeV}$. We showed that all the collected data for symmetric and asymmetric collisions can be described with one universal wounded quark (diquark) emission function. It is not at all obvious that the most (or one of the most) minimalistic model can successfully describe the rapidity distributions for very different systems. Our results indicate that the particle multiplicity at $200 \mathrm{GeV}$ is simply governed by the number of wounded quarks (diquarks) and all other possible effects (e.g., multiple scatterings) are probably less important. Hopefully, our work will trigger other researches to test various models of particle production with all available data.

Below is a more detailed list of our conclusions and comments.

(i) Using the wounded nucleon, quark, and quarkdiquark models we calculated three different wounded source emission functions based on the PHOBOS min-bias $d+{ }^{197} \mathrm{Au}$ data at $\sqrt{s_{N N}}=$ $200 \mathrm{GeV}$ [20].

TABLE X. Same as Table VII but for ${ }^{238} \mathrm{U}+{ }^{238} \mathrm{U}$ collisions.

\begin{tabular}{lcccccc}
\hline \hline & min-bias & $0-5 \%$ & $5-10 \%$ & $15-20 \%$ & $25-30 \%$ & $35-40 \%$ \\
\hline WNM & 62.0 & 211.5 & 182.1 & 131.6 & 93.6 & 64.3 \\
WQDM & 104.5 & 389.1 & 324.8 & 225.2 & 154.6 & 102.0 \\
WQM & 146.8 & 563.4 & 464.6 & 319.7 & 216.1 & 140.5 \\
\hline \hline
\end{tabular}




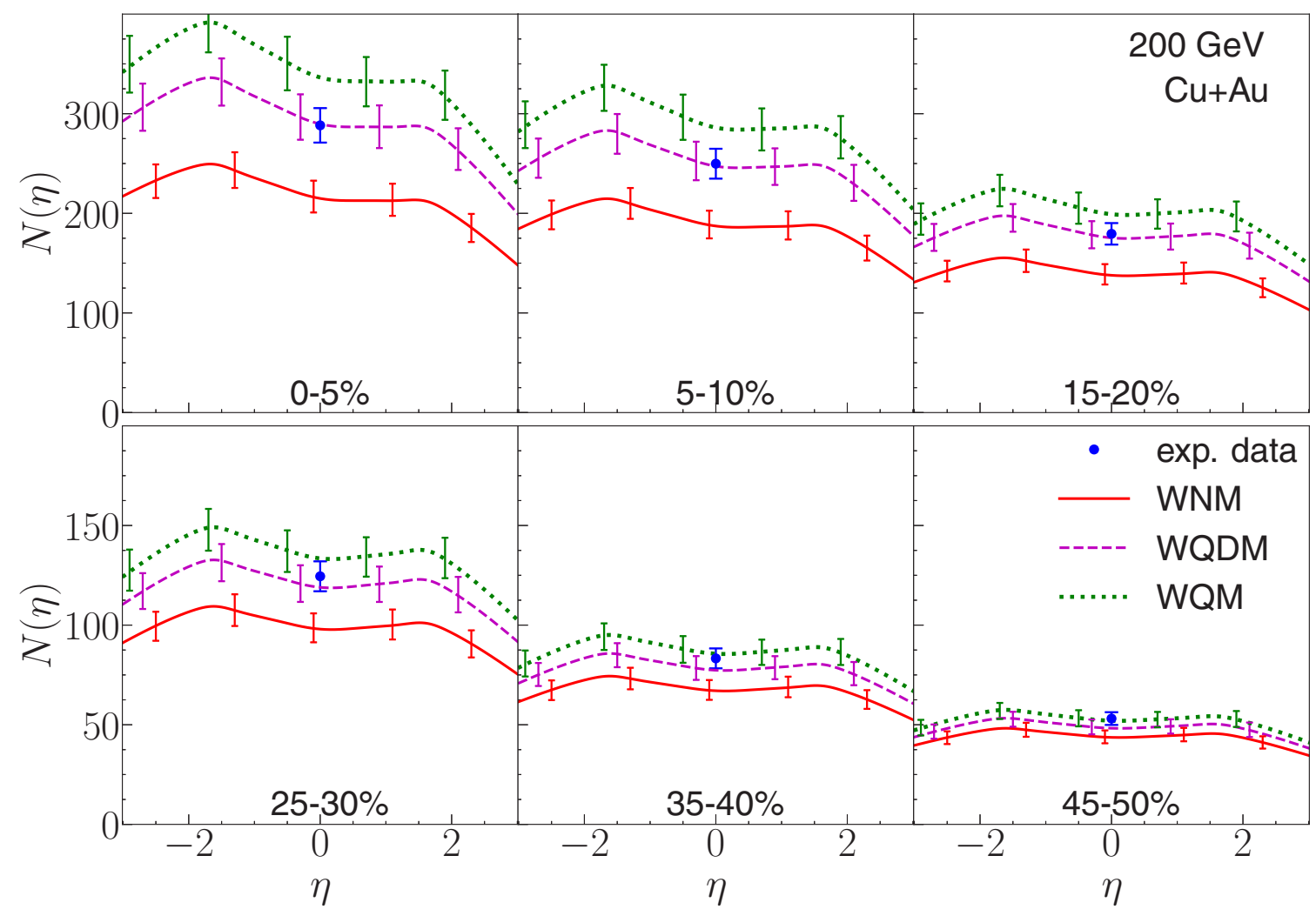

FIG. 8. Same as Fig. 2 but for ${ }^{63} \mathrm{Cu}+{ }^{197} \mathrm{Au}$ collisions. The dots at $\eta=0$ represent the PHENIX data [4].

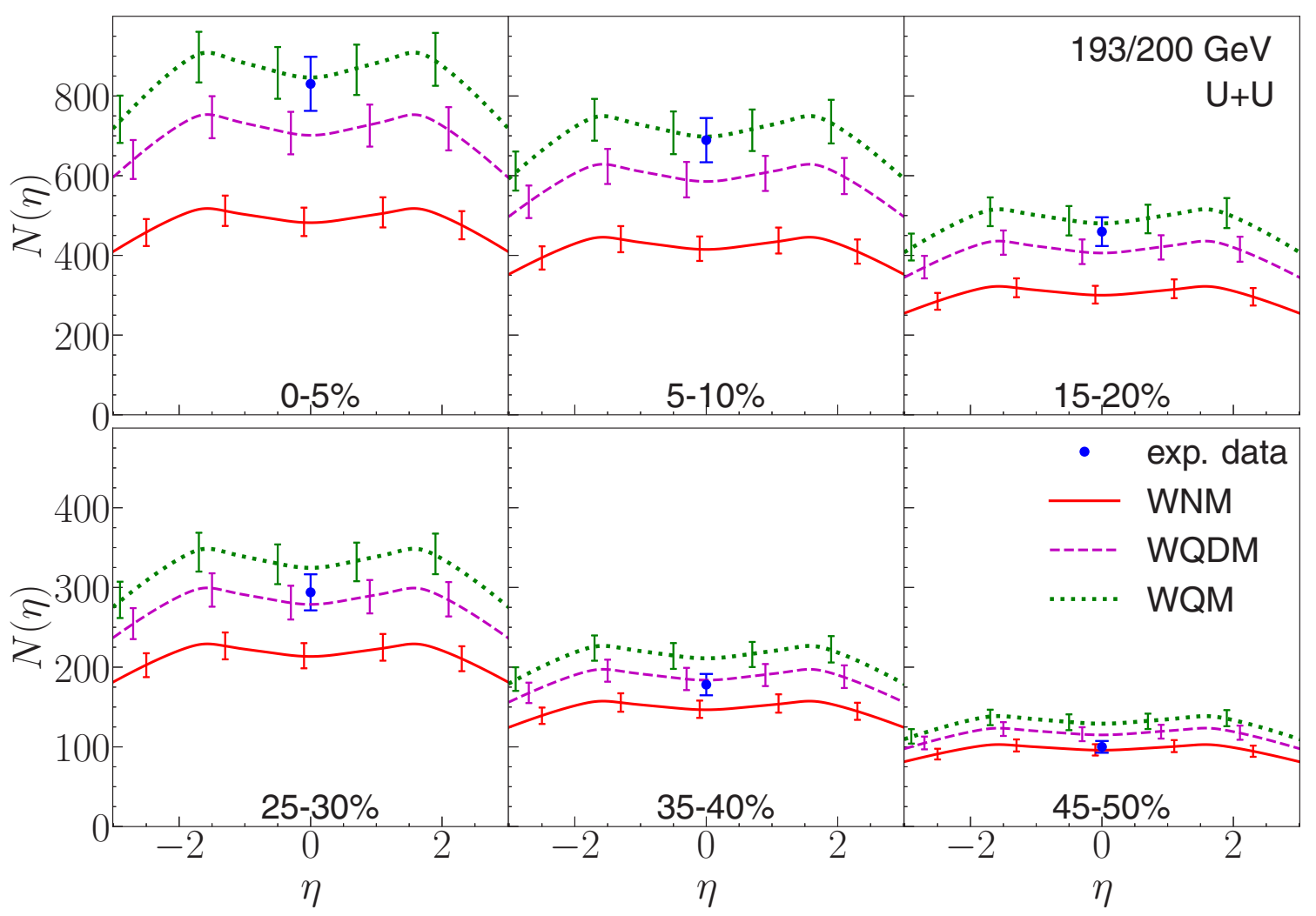

FIG. 9. Same as Fig. 2 but for ${ }^{238} \mathrm{U}+{ }^{238} \mathrm{U}$ collisions. The dots at $\eta=0$ represent the PHENIX data at $\sqrt{s_{N N}}=193 \mathrm{GeV}$ [4]. 


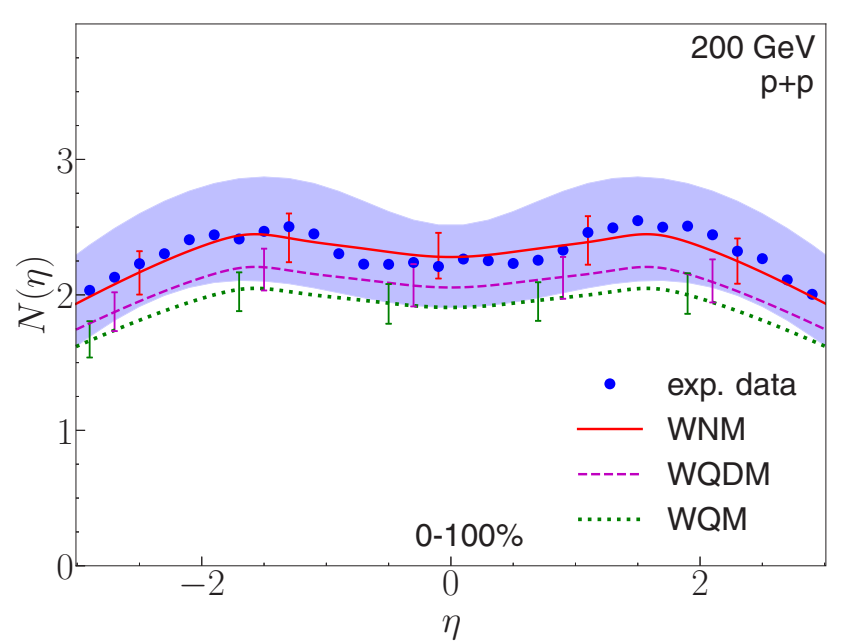

FIG. 10. Same as Fig. 2 but for proton-proton collisions. Dots represent the PHOBOS data [31].

(ii) Using the min-bias emission functions we calculated the pseudorapidity charged particle multiplicity distributions for $p+p, p+{ }^{27} \mathrm{Al}$, $p+{ }^{197} \mathrm{Au}, d+{ }^{197} \mathrm{Au},{ }^{3} \mathrm{He}+{ }^{197} \mathrm{Au},{ }^{63} \mathrm{Cu}+{ }^{63} \mathrm{Cu}$, ${ }^{63} \mathrm{Cu}+{ }^{197} \mathrm{Au}, \quad{ }^{197} \mathrm{Au}+{ }^{197} \mathrm{Au}$, and ${ }^{238} \mathrm{U}+{ }^{238} \mathrm{U}$ collisions at $\sqrt{s_{N N}}=200 \mathrm{GeV}$ in various centrality classes. We note that once the min-bias emission function is known, the rest of our calculation is essentially free of any adjustable parameters.

(iii) All three considered models are in good agreement with the PHENIX data for highly asymmetric collisions. This is not surprising since for these collisions there is only a limited number of nucleons that collide multiple times.

(iv) Results for symmetric collisions of heavy nuclei such as ${ }^{63} \mathrm{Cu}+{ }^{63} \mathrm{Cu}$ and ${ }^{197} \mathrm{Au}+{ }^{197} \mathrm{Au}$ differ significantly among the models. Both wounded quark and quark-diquark models are in quite good agreement with the PHOBOS data. As expected, the wounded nucleon model underpredicts the data, except for very peripheral ones. For $p+p$ interactions, all three models are acceptable.

(v) The wounded quark and quark-diquark models are in quite good agreement with the PHENIX data [4] on ${ }^{63} \mathrm{Cu}+{ }^{197} \mathrm{Au}$ and ${ }^{238} \mathrm{U}+{ }^{238} \mathrm{U}$ collisions. Here we could compare only at $\eta=0$, and hopefully our predictions in the wider range of $\eta$ will be verified experimentally.

(vi) Currently, we are working on extending these models to include the fragmentation regions. This requires considering the unwounded sources (within wounded nucleons) of charged particles [14]. Moreover, it would be also desired to test the discussed models at various collision energies. The wounded quark (diquark) emission function obviously evolves with energy. However, in the first approximation, we expect, e.g., the width of $F(\eta)$ to simply scale with a factor of $\ln (s)$, which is the beam rapidity.

(vii) It would be interesting to confront the recent PHENIX data [21] with different (and much more sophisticated) models of particle production, such as [32-36]. In particular, it would be important to test the color glass condensate inspired models [37-40].

(viii) Usually in relativistic hydrodynamic simulations of heavy ions the wounded nucleon model together with the number of collisions, $N_{\text {coll }}$, component is used to successfully reproduce particle multiplicities [41] for various centralities. In the wounded quark or quark-diquark models the more collisions a nucleon undergoes, the more likely it is to have more wounded constituents and the more particles are produced. Consequently, there is no need to add the $N_{\text {coll }}$ component. It would be interesting to find a clear way to distinguish between the two approaches. Recently, the STAR Collaboration presented the data [42] on elliptic flow vs multiplicity in $\mathrm{U}+\mathrm{U}$ collisions and concluded that the data are better described by wounded quark models.

\section{ACKNOWLEDGMENTS}

This work was partially supported by the Ministry of Science and Higher Education and by the National Science Centre, Grant No. 2018/30/Q/ST2/00101.

\section{APPENDIX}

In order to make the experimental input more convenient, we made fits to the PHOBOS data for $d+{ }^{197} \mathrm{Au}$ collisions [20]. Two independent functions are fitted to the symmetrized and antisymmetrized PHOBOS data. As seen in Fig. 11(a), the antisymmetrized case, $N^{-}(\eta) \equiv N(\eta)-N(-\eta)$, in a considered pseudorapidity range $\eta \in[-3,3]$, is clearly a linear function of $\eta$, that is $N^{-}(\eta)=c \eta$. In the symmetrized case, $N^{+}(\eta) \equiv N(\eta)+N(-\eta)$, the resulting points are naturally resembling a normal distribution in rapidity transformed to pseudorapidity; see Fig. 11(b). Obviously, $N_{\text {fit }}(\eta)=$ $\left[N^{+}(\eta)+N^{-}(\eta)\right] / 2$ resulting in

$$
N_{\text {fit }}(\eta)=\frac{1}{2}\left[A \exp \left(\frac{-y^{2}(\eta)}{2 \sigma_{1}^{2}}\right) \frac{T \cosh \eta}{\sqrt{1+T^{2} \sinh ^{2} \eta}}+c \eta\right],
$$

where $A, \sigma_{1}, c$ are fit parameters, $y$ is rapidity and satisfies $y=$ $\ln \left(\sqrt{1+T^{2} \sinh ^{2} \eta}+T \sinh \eta\right)$, and $T$ is a ratio of transverse momentum to transverse mass and is extracted from the fit. To obtain slightly better fits around $\eta=0$ for the symmetric case, as shown in Fig. 11(b), we multiplied the first term in Eq. (A1) by $1-\alpha \exp \left(\frac{-y^{6}(\eta)}{2 \sigma_{2}^{2}}\right)$, where $\alpha, \sigma_{2}$ are new fit parameters with $\alpha$ much smaller than 1 . The results of this fit differ from that of Eq. (A1) by about one percent (mostly in the $|\eta|<1.5$ region). 

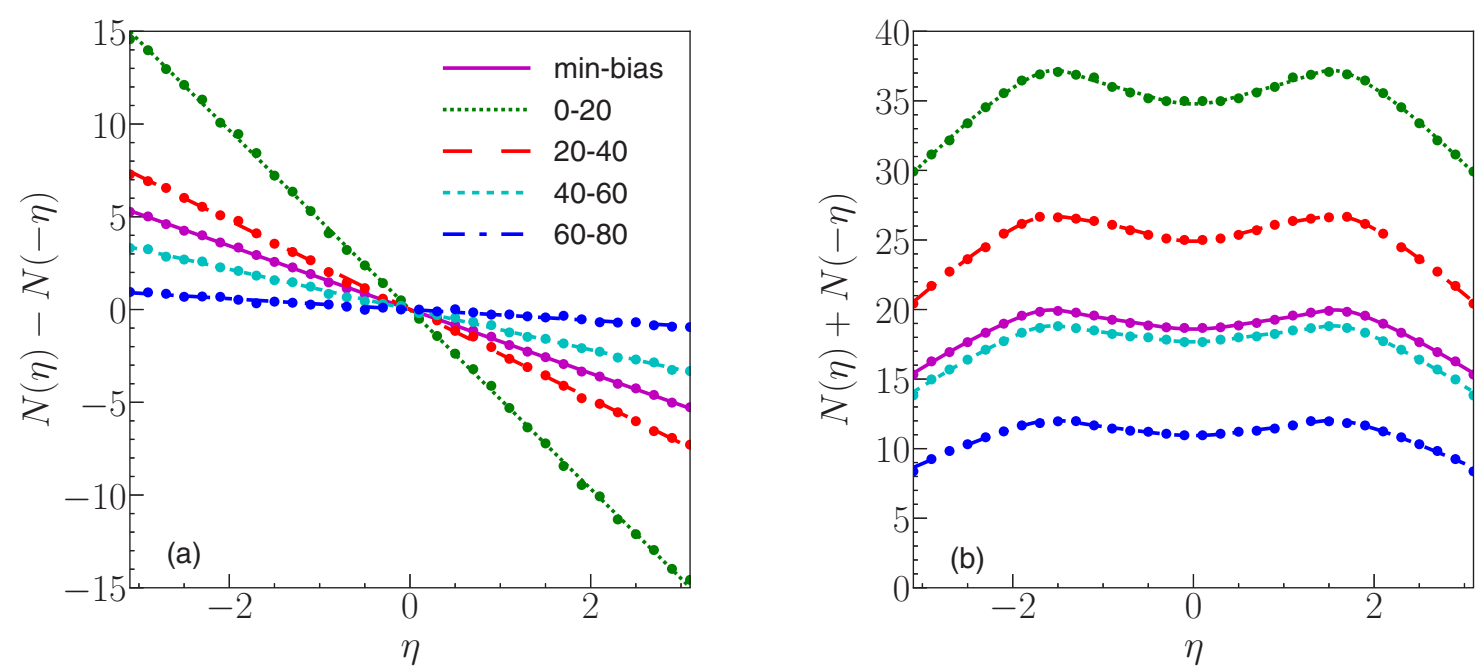

FIG. 11. Fits (lines) to the antisymmetrized (a) and the symmetrized (b) PHOBOS data (dots) for $d+{ }^{197}$ Au collisions in $|\eta|<3$ at $\sqrt{s_{N N}}=200 \mathrm{GeV}[20]$.

[1] A. Bialas, M. Bleszynski, and W. Czyz, Nucl. Phys. B 111, 461 (1976).

[2] A. Bialas, W. Czyz, and W. Furmanski, Acta Phys. Pol. B 8, 585 (1977).

[3] S. S. Adler et al. (PHENIX Collaboration), Phys. Rev. C 89, 044905 (2014).

[4] A. Adare et al. (PHENIX Collaboration), Phys. Rev. C 93, 024901 (2016).

[5] P. Bożek, W. Broniowski, and M. Rybczyński, Phys. Rev. C 94, 014902 (2016).

[6] R. A. Lacey, P. Liu, N. Magdy, M. Csanád, B. Schweid, N. N. Ajitanand, J. Alexander, and R. Pak, Universe 4, 22 (2018).

[7] C. Loizides, Phys. Rev. C 94, 024914 (2016).

[8] J. T. Mitchell, D. V. Perepelitsa, M. J. Tannenbaum, and P. W. Stankus, Phys. Rev. C 93, 054910 (2016).

[9] P. Bożek and W. Broniowski, Phys. Rev. C 96, 014904 (2017).

[10] O. S. K. Chaturvedi, P. K. Srivastava, A. Kumar, and B. K. Singh, Eur. Phys. J. Plus 131, 438 (2016).

[11] L. Zheng and Z. Yin, Eur. Phys. J. A 52, 45 (2016).

[12] M. Barej, A. Bzdak, and P. Gutowski, Phys. Rev. C 97, 034901 (2018).

[13] M. Rohrmoser and W. Broniowski, Phys. Rev. C 99, 024904 (2019).

[14] A. Bialas and A. Bzdak, Phys. Lett. B 649, 263 (2007); 773, 681(E) (2017); Acta Phys. Pol. B 38, 159 (2007); Phys. Rev. C 77, 034908 (2008).

[15] A. Bzdak, Acta Phys. Pol. B 38, 2665 (2007).

[16] F. Nemes and T. Csorgo, Int. J. Mod. Phys. A 27, 1250175 (2012).

[17] T. Csorgo, R. J. Glauber, and F. Nemes, arXiv:1311.2308.

[18] T. Csorgo and F. Nemes, Int. J. Mod. Phys. A 29, 1450019 (2014).

[19] F. Nemes, T. Csörgő, and M. Csanád, Int. J. Mod. Phys. A 30, 1550076 (2015).

[20] B. B. Back et al. (PHOBOS Collaboration), Phys. Rev. C 72, 031901 (2005).

[21] A. Adare et al. (PHENIX Collaboration), Phys. Rev. Lett. 121, 222301 (2018).
[22] B. Alver et al. (PHOBOS Collaboration), Phys. Rev. Lett. 102, 142301 (2009).

[23] B. B. Back et al., Phys. Rev. Lett. 91, 052303 (2003).

[24] A. Bialas and W. Czyz, Acta Phys. Pol. B 36, 905 (2005).

[25] C. Loizides, J. Nagle, and P. Steinberg, SoftwareX 1-2, 13 (2015).

[26] L. Hulthen and M. Sugawara, Structure of Atomic Nuclei/Bau der Atomkerne, Encyclopedia of Physics / Handbuch der Physik (Springer, Berlin, Heidelberg, 1957), Vol. 8/39, pp. 1-143.

[27] J. Carlson and R. Schiavilla, Rev. Mod. Phys. 70, 743 (1998).

[28] H. De Vries, C. W. De Jager, and C. De Vries, At. Data Nucl. Data Tables 36, 495 (1987).

[29] R. Hofstadter, Rev. Mod. Phys. 28, 214 (1956).

[30] R. E. Ansorge et al. (UA5 Collaboration), Z. Phys. C 43, 357 (1989).

[31] B. Alver et al. (PHOBOS Collaboration), Phys. Rev. C 83, 024913 (2011).

[32] S. Jeon and J. I. Kapusta, Phys. Rev. C 56, 468 (1997).

[33] X. N. Wang and M. Gyulassy, Phys. Rev. D 44, 3501 (1991).

[34] M. Bleicher et al., J. Phys. G 25, 1859 (1999).

[35] Z. W. Lin, C. M. Ko, B. A. Li, B. Zhang, and S. Pal, Phys. Rev. C 72, 064901 (2005).

[36] T. Pierog and K. Werner, Nucl. Phys. Proc. Suppl. 196, 102 (2009).

[37] E. Iancu, A. Leonidov, and L. McLerran, arXiv:hep$\mathrm{ph} / 0202270$.

[38] F. Gelis, E. Iancu, J. Jalilian-Marian, and R. Venugopalan, Annu. Rev. Nucl. Part. Sci. 60, 463 (2010).

[39] D. Kharzeev, E. Levin, and M. Nardi, Nucl. Phys. A 730, 448 (2004); 743, 329(E) (2004).

[40] B. Schenke, P. Tribedy, and R. Venugopalan, Phys. Rev. C 89, 024901 (2014).

[41] W. Florkowski, Phenomenology of Ultra-Relativistic Heavy-Ion Collisions (World Scientific, Singapore, 2010).

[42] L. Adamczyk et al. (STAR Collaboration), Phys. Rev. Lett. 115, 222301 (2015). 